\title{
ENSINO A DISTÂNCIA NA EDUCAÇÃO BÁSICA: A INTEGRAÇÃO PEDAGÓGICA DE JOGOS DIGITAIS EM AMBIENTES VIRTUAIS
}

Willian Rochadel ${ }^{1}$ Josi Zanette do Canto ${ }^{2}$

Juarez Bento da Silva ${ }^{3}$ Manuel A. González Delgado ${ }^{4}$ Miguel A. González Rebollo ${ }^{5}$

\section{Resumo}

O uso das tecnologias na educação exige uma adequada integração dos conhecimentos a fim de evitar frustrações e desperdícios de tempo. Logo, um plano de aplicação precisa delinear as reflexões não apenas didáticas, mas também tecnológicas e do conteúdo, uma proposta de gestão do conhecimento relacionada ao TPACK(TechnologicalPedagogical Content Knowledge). Assim, o objetivo desse artigo é apresentar o desenvolvimento de um plano de aplicação de jogos digitais que permita a observação ampla dos conhecimentos necessários para a realização das atividades pedagógicas a distância baseada no TPACK. Desse modo, o plano permite sistematizar a integração dos jogos em uma concepção pedagógica de extensão da sala de aula e ampliação dos conhecimentos expostos. Além disso, a análise em um estudo empírico com uma turma do ensino fundamental permitiu validar o plano. Apesar das limitações em relação as extensões dos jogos disponibilizados, a $\mathrm{EaD}$ se mostrou viável no complemento dos conteúdos.

Palavras-chave: Jogos Educacionais; Ensino de Geografia; Ambientes Virtuais de Aprendizagem; Objetos Educacionais.
$* * *$

1 Mestrando, e-mail: willian.rochadel@ ufsc.br

2 Pós-graduanda, e-mail: josizanettecanto@ hotmail.com

3 Doutor, e-mail: juarez.b.silva@ieee. org

$4 \mathrm{PhD}$, e-mail: manuelgd@termo. uva.es

$5 \mathrm{PhD}$, e-mail: mrebollo@eii.uva.es 


\section{INTRODUÇÃO}

A utilização de jogos educacionais é cada vez mais defendida nos processos pedagógicos pelas características lúdicas de aprendizado (JAPPUR, 2014). Porém, diversos desafios prejudicam a sua utilização nas escolas.

O primeiro limitante é o tempo de aula da Educação Básica. No Brasil, cada aula possui cerca de 50 minutos, que são considerados como uma hora aula. Estimando 200 dias letivos e em média três aulas semanais, são 120 horas aula por ano. Período muitas vezes prejudicado por feriados, paralizações e greves.

\section{OUTRO FATOR É A}

INFRAESTRUTURA DAS ESCOLAS,

AO QUAL É ESTIMADO QUE

$56 \%$ DAS 151.884 ESCOLAS

PÚBLICAS BRASILEIRAS AINDA

NÃO POSSUEM LABORATÓRIOS

DE INFORMÁTICA, SEGUNDO O

Censo Escolar (Inep, 2013).

$\mathrm{E}$, mesmo as instituições que possuem laboratórios de informática, dificilmente dispõem de computadores suficientes para a aplicação individual.

Portanto, (a) ao considerar o tempo utilizado para deslocar uma turma até a sala de informática; (b) permitir o rodízio de estudantes por computador; e (c) não dispor de uma conexão adequada para uso da internet, são questões que prejudicam a integração pedagógica de jogos digitais na escola.

Nesse contexto, uma alternativa é o uso de ambientes virtuais de aprendizagem (AVA). Comumente utilizados na modalidade de
Ensino a Distância, esses ambientes apresentam soluções para o gerenciamento de recursos, conteúdos e atividades on-line (PEREIRA; SCHMITT; DIAS, 2007).

Porém, dispor do recurso não garante o sucesso da aplicação. É necessário considerar um adequado planejamento pedagógico, a fim de integrar os jogos digitais dentro das limitações.

Assim, para sistematizar a integração dos jogos de modo que a aplicação seja realizada fora do período de aula, o artigo apresenta uma concepção pedagógica de extensão da sala de aula e ampliação dos conhecimentos expostos. São descritos então os critérios, as etapas e o processo de criação até a aplicação de um plano de aula voltado ao uso de jogos em um AVA.

Para isso, utiliza a metodologia Design Science Research (PEFFERS et al., 2007) na definição das etapas de organização e na prática mediada a Distância. Como resultado, além das etapas do processo, apresenta um plano de aplicação baseado na concepção de Jappur (2014), relacionando à metodologia TPACK (Technological Pedagogical Content Knowledge) (KOEHLER; MISHRA, 2009).

O artigo é então estruturado em seções também de acordo com os processos da Design Science Research, sendo na seção 2 apresentado o referencial teórico em relação à modalidade de Educação a Distância na Educação Básica e o contexto de aplicação, ou seja, da disciplina de geografia no Ensino Fundamental, seguida por uma explanação da integração de jogos digitais na educação e o conceito de AVA. Na seção 3, são discutidos os processos metodológicos a 
partir da definição da pesquisa e apresentação da solução proposta. Em seguida, na seção 4, é descrito o desenvolvimento e organização, como a definição do jogo, a construção do plano e o plano detalhado. Por fim, são apresentadas as avaliações e resultados na seção 5 .

\section{EDUCAÇÃO A DISTÂNCIA NA EDUCAÇÃO BÁSICA}

No Brasil, a Secretaria de Educação a Distância (SEED) do Ministério da Educação (MEC) é responsável por atuar como "[...] um agente de inovação tecnológica nos processos de ensino e aprendizagem, fomentando a incorporação das Tecnologias de Informação e Comunicação (TICs) e das técnicas de educação a distância aos métodos didático pedagógicos"(MEC, 2009). A SEED é a responsável por programas como oBanco Internacional de Objetos Educacionais (BIOE), o Portal do Professor, o Programa Um Computador por Aluno (PROUCA) e o Programa Banda Larga nas Escolas.

Apesar da SEED ser criada pelo Decreto no 1.917, de 27 de maio de 1996 (BRASIL, 1996), apenas com o decreto no 5.622 , de 19 de dezembro de 2005 (BRASIL, 2005), o Ensino a Distância foi regulamentado em todos os níveis de ensino, mais especificamente pelo Art. ${ }^{\circ} 2$ do referido decreto.

Já para o Ensino Fundamental e Médio, a EaD é apenas admitida para: (a) complementação de aprendizagem; (b) em situações emergenciais do cidadão; ou (c) ministrados por meio de educação especial ou de jovens e adultos, conhecidos como supletivos, conforme o Art. ${ }^{\circ}$ 31 (BRASIL, 2005).

Assim, o planejamento de atividades que requerem a prática mediada a Distância para o Ensino Básico regular precisam ser consideras como uma "complementação de aprendizagem".
A partir dessa delimitação, a pesquisa aqui apresentada planeja tratar as atividades a Distância como um complemento e auxílio aos estudantes, em especial na disciplina de geografia, no Ensino Fundamental.

\subsection{A disciplina de Geografia no Ensino Fundamental}

O ensino de geografia tem como objetivo tornar o aluno capaz de compreender a relação do homem com o meio, assimilar que dessa relação surge o espaço em que a humanidade vive, o espaço geográfico, que é o resultado de ações históricas e sociais formadas pelo grupo de elementos naturais e objetos produzidos pelo homem, em escalas que variam do local para o global (PEREIRA, 2012).

Os Parâmetros Curriculares Nacionais (BRASIL,1997) definem os princípios da disciplina de geografia no Ensino Fundamental em:

\footnotetext{
- Conhecer e valorizar a pluralidade do patrimônio sociocultural brasileiro, bem como aspectos socioculturais de outros povos e nações, posicionando-se contra qualquer discriminação baseada em diferenças culturais, de classe social, de crenças, de sexo, de etnia ou outras características individuais e sociais;

- Utilizar as diferentes linguagens verbais, musical, matemática, gráfica, plástica e corporal como meio para produzir, expressar e comunicar suas
} 
ideias, interpretar e usufruir das produções culturais, em contextos públicos e privados, atendendo a diferentes intenções e situações de comunicação;

- Saber utilizar diferentes fontes de informação e recursos tecnológicos para adquirir e construir conhecimentos;

- Questionar a realidade formulando-se problemas e tratando de resolvê-los, utilizando para isso o pensamento lógico, a criatividade, a intuição, a capacidade de análise crítica, selecionando procedimentos e verificando sua adequação.

Portanto, compreender o ambiente e o contexto do espaço é fundamental para o desenvolvimento da interpretação, ciente das questões sociais, ambientais e econômicas.

\section{LOGO, O ENSINO DE GEOGRAfIA}

MOSTRA A SUA IMPORTÂNCIA

PARA O DESENVOLVIMENTO SO-

CIAL E CRÍTICO DO ESTUDANTE,

AMPLIADO PELA TECNOLOGIA

NA CONSTRUÇÃO DESSE CO-

NHECIMENTO EM UM PROCESSO

QUE O ENVOLVA E SE SINTA

INCLUÍDO COMO CIDADÃO NA

ERA DO CONHECIMENTO, QUE,

PELO RÁPIDO ACESSO À INFOR-

MAÇÃO E A DISPONIBILIZAÇÃO

DE DADOS MUNDIAIS, PERMITE

UMA ASSOCIAÇÃO DOS SABERES

(GADOTTI, 2000).

Para Cavalcanti (2010), o objetivo de ensinar a geografia para crianças e jovens é auxiliá-los a perceber o mundo, através de raciocínios e concepções mais articuladas e aprofundadas a respeito do espaço que os mesmos habitam. Auxilia os discentes a serem cidadãos mais ativos, criativos, preparados para as exigências do mundo atual, olhando geograficamente para as mudanças dos novos tempos, para que os mesmos possam, partindo de seus conhecimentos adquiridos, posicionar-se criticamente frente às inovações das sociedades.

\subsection{Jogos na Educação}

O entretenimento dos jogos sempre chamou a atenção de usuários em diferentes propósitos. Um jogo pode ser definido como uma atividade realizada dentro de um limite espaço-temporal com um fim em si mesmo, ao qual segue regras especificamente definidas (HUIZINGA, 2014). A função é então a representação de uma realidade recriada pela metáfora (ROZA, 1996). Portanto, jogos são atividades conduzidas em um contexto de realidade imaginada, onde os participantes possuem uma meta a ser realizada de acordo com regras estabelecidas (THOMPSON; BERBANK-GREEN; CUSWORTH , 2007).

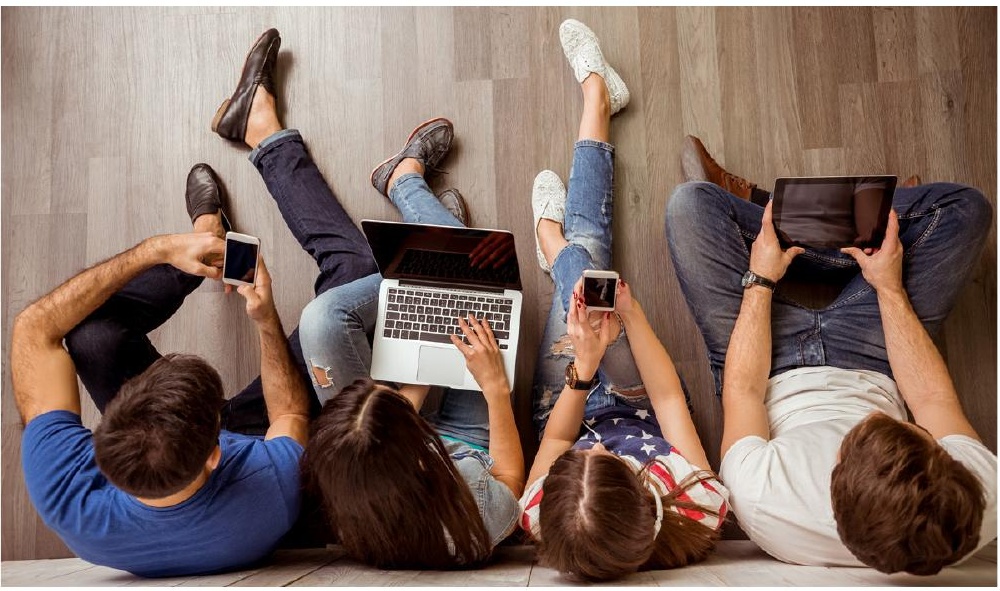

Essa atividade remete a um passado longínquo, encontrada desde os primeiros registros do homem. Os vestígios de um jogo egípcio denominado "Senet" é uma das mais antigas evidências arqueológicas disso, datado em 3.500 anos antes de Cristo. Mas, foi a partir do desenvolvimento da eletrônica que os jogos tomaram formatos 
digitais, ganhando cada vez mais adeptos à medida que a tecnologia se amplia.

A partir da década de 50, com os primeiros computadores, alguns jogos tomaram um formato eletrônico. Segundo Salen e Zimmerman (2012), esse formato mantém as características de jogabilidade, porém com adicionais como: (1) Interatividade imediata, mas restrita; (2) Manipulação da informação; (3) Sistemas complexos e automatizados; (4) Comunicação em rede.

Já a concepção de jogos digitais na educação converge a área pedagógica e tecnológica em um ambiente lúdico como auxílio ao processo de ensino e aprendizagem.

Segundo Jappur (2014), os jogos potencializam a interatividade, a colaboração e a aprendizagem. Portanto, possibilitam uma aprendizagem baseada na experiência (experience-based learning), além de apoiar o desenvolvimento de pensamentos complexos, como análises e aplicação de conhecimentos, resultando em um aumento do nível de retenção do que foi estudado (SILVA, 2012).

O desenvolvimento de jogos para a educação ganha cada vez mais o incentivo do governo e de empresas que mantém repositórios de objetos de aprendizagem na internet de livre acesso, em sua maioria (MEC, 2015). Porém, além da disponibilização, é necessária uma integração planejada pedagogicamente.

Portanto, é imprescindível a atuação constante do docente na adaptação e uso de jogos ligados à disciplina, ao conteúdo e às possibilidades tecnológicas de acesso.

Essa integração encontra desafios interdisciplinares a serem explorados. As alternativas de inclusão no processo pedagógico exigem um estudo empírico e observações constantes de melhorias, para não se tornarem um contratempo ou uma decepção, tanto aos docentes como estudantes.

Logo, a importância dessa pesquisa se justifica pelo alinhamento do trabalho colaborativo entre educador, colaborador técnico e pedagógico. Machado, Reis e Bentes (2015) trazem uma discussão semelhante sobre o uso de jogos educacionais mediados a Distância e que envolve a temática das redes sociais. Porém, o foco é um relato de experiência pelo grupo de gestores de uma universidade e baseia a metodologia de Ensino a Distância a partir da concepção do método do action learning.

\subsection{Ambientes Virtuais de Aprendizagem}

Há diversos sistemas comuns de compartilhamento de conteúdo que podem ser utilizados gratuitamente na web, como fóruns, redes sociais, mensagens eletrônicas, blogs, sites e discos virtuais. Porém, os sistemas especializados se diferem de sistemas comuns pela especificidade e foco no conteúdo. No caso da educação, os Ambientes Virtuais de Aprendizagem (AVA) são os sistemas adequados para mediar o processo de ensino-aprendizagem a Distância (PEREIRA et al., 2007). Entre os mais utilizados para essa modalidade está o Moodle.

O Moodle ${ }^{1}$ (ModularObject-Oriented Dynamic Learning Environment) permite o desenvolvimento de sites web dinâmicos, o compartilhamento de materiais didáticos, além de recursos, como chat,fórum e atividades personalizadas em diferentes modos. Ainda possui características de um sistema de gestão para avaliação dos alunos, controle de acesso e suporte pedagógico.

${ }^{1}$ Disponível em: https://moodle.org/ 
Por ser gratuito, modular e ser mantido por uma extensa comunidade de desenvolvedores, $\mathrm{o}$ sistema é amplamente utilizado, em especial,na Educação a Distância e também como auxílio às instituições de Ensino Superior. Porém, na Educação Básica, o uso não é comum, devido à necessidade de uma infraestrutura de servidores e internet, que são recursos deficientes na maioria das instituições de ensino.

A importância do compartilhamento de materiais complementares nestes ambientes traz novas alternativas de estudo para que o docente disponibilize o conteúdo de sala de aula, ou mesmo recursos extras para incremento da aprendizagem. Logo, essas possibilidades podem ser melhor exploradas também na educação básica, até como um ambiente cooperativo de compartilhamento e discussão. $\mathrm{Na}$ abordagem desse artigo, o Moodle servirá como uma extensão da sala de aula e uma alternativa para o Ensino a Distância, devido ao curto tempo do período letivo.

\section{PROCESSO METODOLÓGICO}

\subsection{Definição da Pesquisa}

O propósito desta pesquisa é apresentar um processo de desenvolvimento de planos de aula voltados ao compartilhamento de conhecimento através da integração de jogos em AVA para a Educação Básica.

A importância desta pesquisa se justifica pela carência tecnológica das escolas e o curto tempo de aula que prejudicam a aplicação de jogos no período letivo. Define-se então a questão de pesquisa em: Quais critérios são necessários para integrar o uso de jogos em AVA na Educação Básica?

Para isso, são definidos como objetivos específicos:

- descrever o processo de desenvolvimento de um plano de aula;

- desenvolver um plano de aplicação de jogos;

- integrar o plano de aplicação ao plano de aula;

- avaliar o uso do plano.

\subsection{Planejamento da aplicação}

O desenvolvimento de planos de aula faz parte da prática diária dos docentes.Um plano de aula orienta a preparação e as entregas de um período, sistematizando as atividades de interação entre professor e estudante. Portanto, planos de aula ajudam a delinear:

\section{$[\ldots]$ o que os alunos devem aprender (objetivos de aprendizagem, o assunto), como o processo de ensino e aprendizagem será organizado (atividades de aprendizagem, abordagem de ensino), e quais os recursos serão necessários (materiais de estudo, tecnologia). (JANSSEN; LAZONDER, 2015).}

A partir desse planejamento da aula, um outro plano organiza e descreve os procedimentos que serão tomados na ação pedagógica de aplicação do recurso (MURTHY; IYES; WARRIEM, 2015). 
A proposta inicial do plano é a integração dos jogos digitais em ambientes virtuais a fim de que os próprios estudantes tomem a iniciativa de utilizá-los. Assim, poderão realizar a atividade no momento mais propício, sem consumir o tempo da aula.

Para Jappur (2014), o plano de aplicação é adaptado a partir da metodologia TPACK, a fim de integrar as relações dos conhecimentos tecnológicos, pedagógicos e de conteúdo na aplicação de jogos.

\section{O Technological Pedagogical Content Knowledge} (TPACK, ou Conhecimento Tecnológico Pedagógico de Conteúdo ou Disciplinar) refere-se aos conhecimentos que o docente necessita para usar estrategicamente a tecnologia para o ensino.

Nessa metodologia, os conhecimentos tecnológicos, pedagógicos e de conteúdo das disciplinas são convergidos para compreender e desenvolver práticas que abordem o ensino e a aprendizagem das disciplinas com a integração das novas tecnologias da informação e comunicação (KOEHLER;

MISHRA, 2009).
A metodologia é baseada na ideia do Conhecimento Pedagógico do Conteúdo (PCK ou PACK) proposta por Shulman (1987) e, a partir da inclusão do conhecimento tecnológico, proposta por Koehler e Mishra (2009), a fim de possibilitar aos educadores e pesquisadores desenvolver planejamentos mais elaborados no uso das tecnologias.

O modelo TPACK inclui a integração entre conteúdo, pedagogia e tecnologia e os principais componentes do conhecimento: conteúdo ou disciplinar (CK), pedagogia (PK) e tecnologia (TK), que se superpõem e geram quatro novas formas de conteúdo inter-relacionado (TPK, TCK e PCK), que se cruzam no centro para formar o TPACK (KOEHLER; MISHRA, 2009), conforme demonstrado na Figura 1.

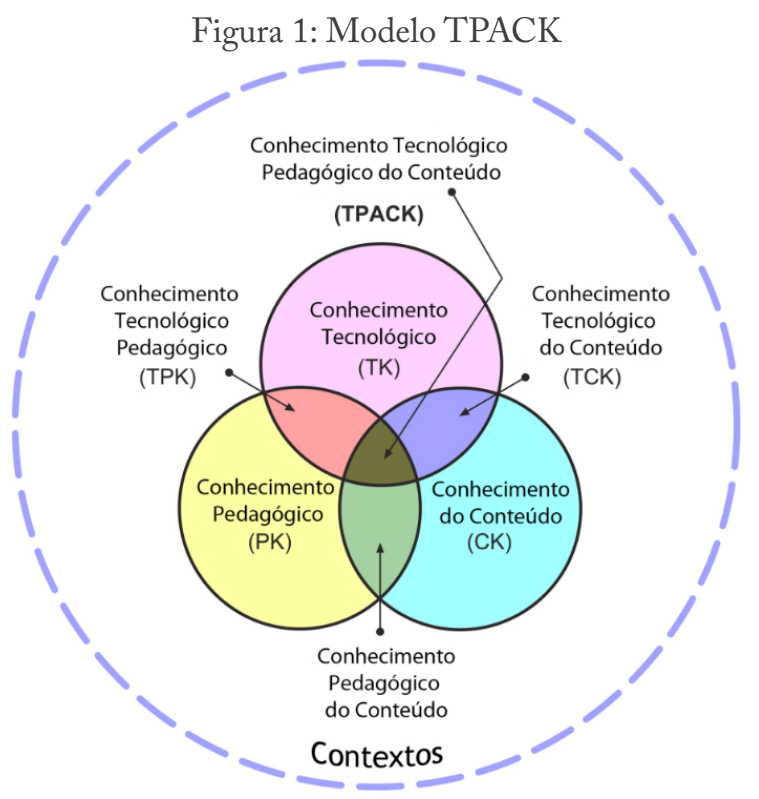

Fonte: Koehler e Mishra (2009) 
São competências do TPACK na implementação e utilização do AVA para o ensino virtual das disciplinas: a representação de ideias mediante ouso de tecnologia; as técnicas pedagógicas que utilizam a tecnologia de formas construtivas para ensinar um conteúdo; o conhecimento sobre o que torna fácil ou difícil a compreensão de um conceito e como a tecnologia pode contribuir para compensar estas dificuldades que os alunos enfrentam; o conhecimento dos conhecimentos prévios dos alunos e de como a tecnologia pode ser utilizada para construir $\mathrm{co}^{-}$ nhecimento interdisciplinar (ALEXANDRE et al., 2014).

\subsection{Estratégia de desenvolvimento}

O desenvolvimento do plano de aula se constitui na definição do conteúdo; planejamento da intervenção pedagógica e avaliação diagnóstica; pesquisa de recursos; definição da explanação do conteúdo e da forma de avaliação (JANSSEN; LAZONDER, 2015). Os planos de ação organizam a integração dos recursos, práticas e caracterizações. Nesta pesquisa, o plano de ação é desenvolvido sobre a necessidade da aplicação.

A principal estratégia de aplicação discutida neste artigo está na sistematização do plano de ação relacionado aos jogos digitais. Para isso, segue a metodologia Design Science Research, que cria e avalia artefatos destinados à resolução de problemas, segundo a demanda de contribuir, examinar e comunicar resultados da pesquisa (VON ALAN et al., 2004).

A Metodologia de Pesquisa da Ciência do Design (Design Science Research Methodology - DSRM) engloba princípios, práticas e procedimentos para a realização de pesquisas com resultados em inovações sociais e técnicas em recursos de informação em uma solução integrada (PEFFERS et al., 2007), conforme a Figura 2.

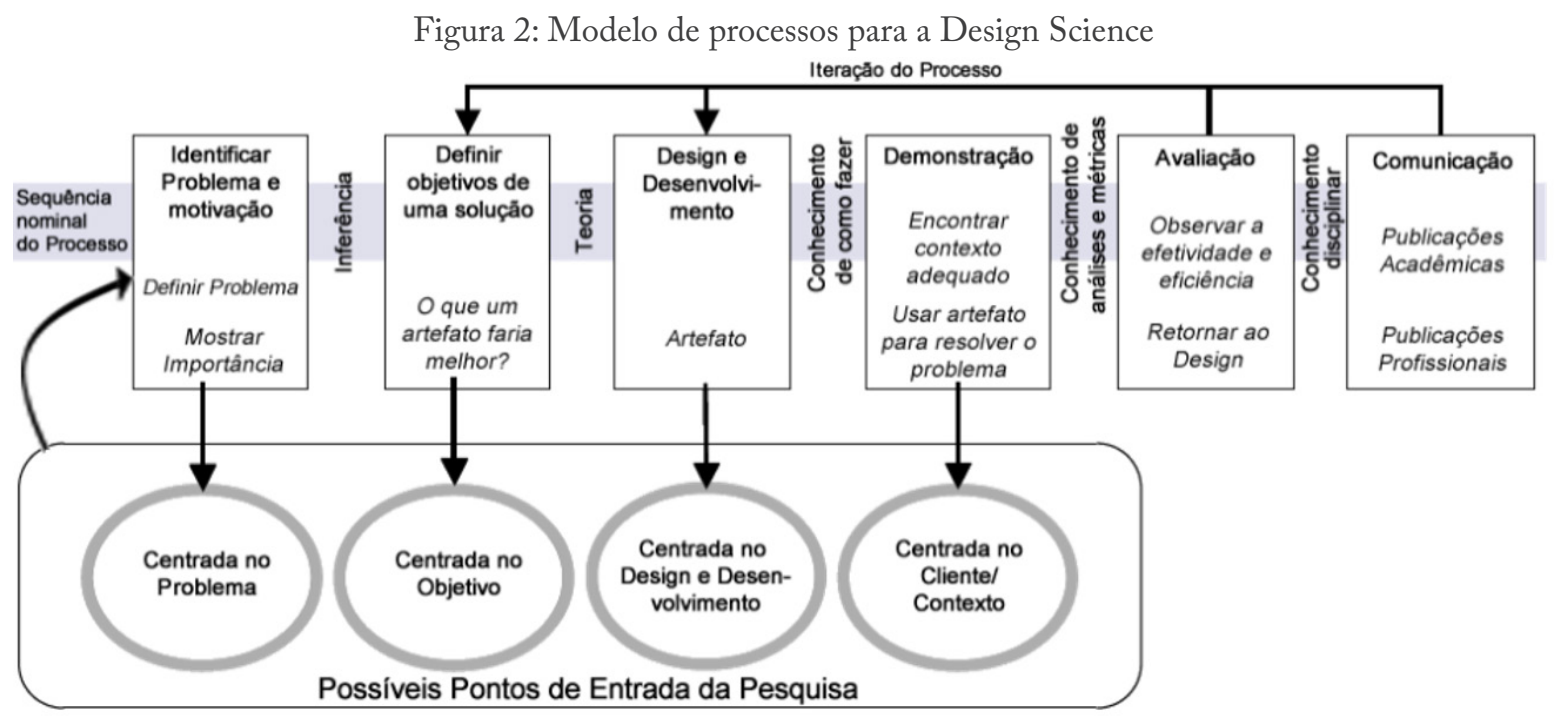

Fonte: Traduzido de Peffers et al. (2007) 
Portanto, a metodologia se constitui aqui em um arcabouço teórico para fundamentar as ações pedagógicas e relacioná-las aos estudos do TPACK.A partir disso,é possível identificar competências e necessidades do docente na aplicação, a fim de organizar e buscar alternativas que o auxiliem na execução. É esperado, assim, que os aspectos pedagógicos, tecnológicos e do conteúdo se complementem em sua prática educacional.

\section{DESENVOLVIMENTO E ORGANIZAÇÃO}

A ação foi aplicada em uma escola pública brasileira da Região Sul de Santa Catarina com 637 estudantes. A instituição de ensino possui uma sala de informática com cinco computadores, que utilizam o Sistema Operacional Linux Educacional 3.0. Cada computador é ainda dividido para dois monitores em áreas de trabalho individuais, somando 10 estações de trabalho, e outros dois computadores estavam parados sem manutenção.

A docente que participou do desenvolvimento possui 12 anos de atuação como educadora no Ensino Fundamental e Médio. Leciona aulas de geografia em duas escolas públicas e em uma instituição particular do município, com carga horária total de 40 horas semanais. A turma do $9^{\circ}$ ano do Ensino Fundamental II foi escolhida pela docente devido ao histórico de participação em outras atividades em seus dois anos de atuação com o grupo.

\section{EM VIRTUDE DA CARÊNCIA}

DE INFRAESTRUTURA NA

ESCOLA, O ESPAÇO NO

MoOdLE FOI DISPONIBILIZADO

PELO LABORATÓRIO DE

EXPERIMENTAÇÃO REMOTA (REXLAB) da Universidade Federal de Santa Catarina (UFSC) - Campus Araranguá. O curso foi montado no ambiente com materiais didáticos do próprio docente da disciplina de geografia e os estudantes da turma foram cadastrados com usuários individuais.

Assim, considerando as definições metodológicas e referencial teórico da seção anterior, serão apresentados, nos tópicos, a seguir, o desenvolvimento do plano, a definição e a aplicação dos jogos.

\subsection{O plano de aplicação}

A partir da proposta de Jappur (2014), o plano de aplicação foi construído e preenchido adaptando-se à necessidade do contexto pela docente. Nessa observação da concepção, foram acrescentados requisitos específicos do uso esperado da tecnologia e as necessidades do AVA. Para isso, foi relacionada à metodologia TPACK na elaboração do plano com a docente.

Por fim, foi concebido um plano que organiza o processo pedagógico relacionando os dispositivos e ferramentas necessários ao conteúdo, além da proposta de avaliação, considerando, assim, as necessidades tecnológicas, pedagógicas e do conteúdo, conforme apresentado no tópico 4.1. 
A estratégia de aplicação não possui um ponto determinado como início. Afinal, assim como o assunto de uma matéria pode originar a necessidade de um jogo, um jogo também pode despertar o interesse de uso. Todos esses itens são pensados a partir do plano de aula e voltado sobre o jogo em si. Por fim, o plano de aplicação compõe o plano de aula. Então, as etapas se dão de maneira sequencial, iterativas e interativas, construindo um modelo de plano para reflexão da ação.

É importante ressaltar que todos esses requisitos, sem o prévio planejamento, acabam por frustrar a aplicação. Para melhor definição dos trechos, o plano foi organizado em duas partes, conforme os tópicos a seguir.

\subsubsection{Identificação e definição dos conhecimentos tecnológicos, pedagógicos e de conteúdo}

A parte I do plano está voltada à identificação do plano e à relação dos conhecimentos caracterizados em três tópicos. Nessa parte, o professor define previamente os conhecimentos necessários para o plano e aplicação, além de especificar as tecnologias (Quadro 1).
I. Identificação: neste tópico, constam os dados referente à identificação da disciplina, turma, participantes e o período de disponibilização. Essa parte é essencial para a organização e a integração do plano de aula e o ensino.

II. Conhecimentos na ação: relaciona os conhecimentos pedagógicos, tecnológicos e do conteúdo necessários com a participação de cada integrante. $\mathrm{O}$ objetivo é então prever as condições e responsabilidades, o que colabora para que o docente tenha uma maior confiança na aplicação.

\section{Requisitos e conhecimentos tecno-} lógicos: apresenta dados referentes aos dispositivos que serão utilizados, a necessidade de conta ou senha, o tipo do jogo e o formato do jogo ou extensão, como Flash, HTML 5, Java. É um tópico com certa complexidade tecnológica, porém fundamental para definir a plataforma em que o jogo será disponibilizado e então saber descrever a atividade para os participantes.

IV. Conhecimentos do conteúdo: define os conteúdos que serão discutidos, além de permitir a organização dos conhecimentos prévios que o estudante precisará estudar para realizar a atividade. 


\section{PLANO DA APLICAÇÃO}

\begin{tabular}{|c|c|c|c|}
\hline $\begin{array}{l}\text { Curso/disciplina/ } \\
\text { série: }\end{array}$ & $\begin{array}{l}\text { Geografia } / 9^{\circ} \text { ano } \\
\text { Ensino Fundamental II }\end{array}$ & $\begin{array}{l}9^{\circ} \text { ano Ensino } \\
\text { Fundamental II }\end{array}$ & $\begin{array}{l}\text { Movimentos } \\
\text { da Terra }\end{array}$ \\
\hline Participantes: & $\begin{array}{l}\text { Docente, discentes, } \\
\text { colaborador }\end{array}$ & Período de disponibilização & $08-16 / 06 / 2015$ \\
\hline \multirow{3}{*}{$\begin{array}{l}\text { Conhecimentos } \\
\text { na ação }\end{array}$} & Pedagógico & Tecnológico & Conteúdo \\
\hline & Professor & $\begin{array}{c}\text { Professor, estudantes e } \\
\text { colaborador }\end{array}$ & Professor \\
\hline & & Fórum & \\
\hline \multirow{4}{*}{$\begin{array}{l}\text { Requisitos e } \\
\text { conhecimentos } \\
\text { Tecnológicos }\end{array}$} & Dispositivo & \multicolumn{2}{|c|}{ Computador: devido a extensão dos jogos. } \\
\hline & Conta do usuário & \multicolumn{2}{|l|}{ Moodle } \\
\hline & Formato do Jogo & \multicolumn{2}{|l|}{ Flash / HTML } \\
\hline & Tipo do Jogo & \multicolumn{2}{|c|}{ Charadas / Questionário online } \\
\hline \multirow{2}{*}{$\begin{array}{l}\text { Conhecimentos } \\
\text { do conteúdo }\end{array}$} & \multicolumn{3}{|l|}{ Conteúdo: } \\
\hline & \multicolumn{3}{|c|}{ Movimentos da terra, orientação e localização no espaço geográfico. } \\
\hline
\end{tabular}

Fonte: Dos autores (2016)

\subsubsection{Reflexão da Ação}

A parte II do plano está relacionada à reflexão prévia e posteriormente à aplicação (Quadro 2). Nessa parte, o docente pondera aspectos específicos ao encaminhamento pedagógico e estratégias que serão utilizadas, desde a apresentação até a avaliação.

\section{Reflexão para a ação pedagógica: nesse} bloco, são especificados o objetivo pedagógico de utilização; a modalidade de apresentação do conteúdo, do plano, do jogo e da avaliação; as estratégias pedagógicas para a aprendizagem; o cronograma do conteúdo, a aplicação e a avaliação especificados de acordo com a modalidade em sala ou a Distância; os indicadores de aprendizagem; e os instrumentos de avaliação.

II. Reflexão na ação: descreve os elementos considerados relevantes durante a ação, ou seja, busca relacionar pontos de avaliação previamente pensados e, posteriormente, ao processo realizado.

Considerando o caráter novo do plano de aplicação, a construção foi conjunta entre docente e pesquisadores. A versão desenvolvida já apresenta uma simplificação de pontos que se tornaram complexos ou dispensáveis ao docente. Dentre as dificuldades constatadas, as definições tecnológicas foram as mais destacadas, o que reforça a necessidade de uma organização conforme apresentada. 
O documento padrão do docente e da instituição de ensino trazem o planejamento de todos os conteúdos, recursos e as avaliações bimestrais definidas. Portanto, a definição da aplicação foi apenas mencionada no plano de aula completo, sendo o plano de aplicação inserido como um documento extra.

Quadro 2: Plano de Aplicação Parte II

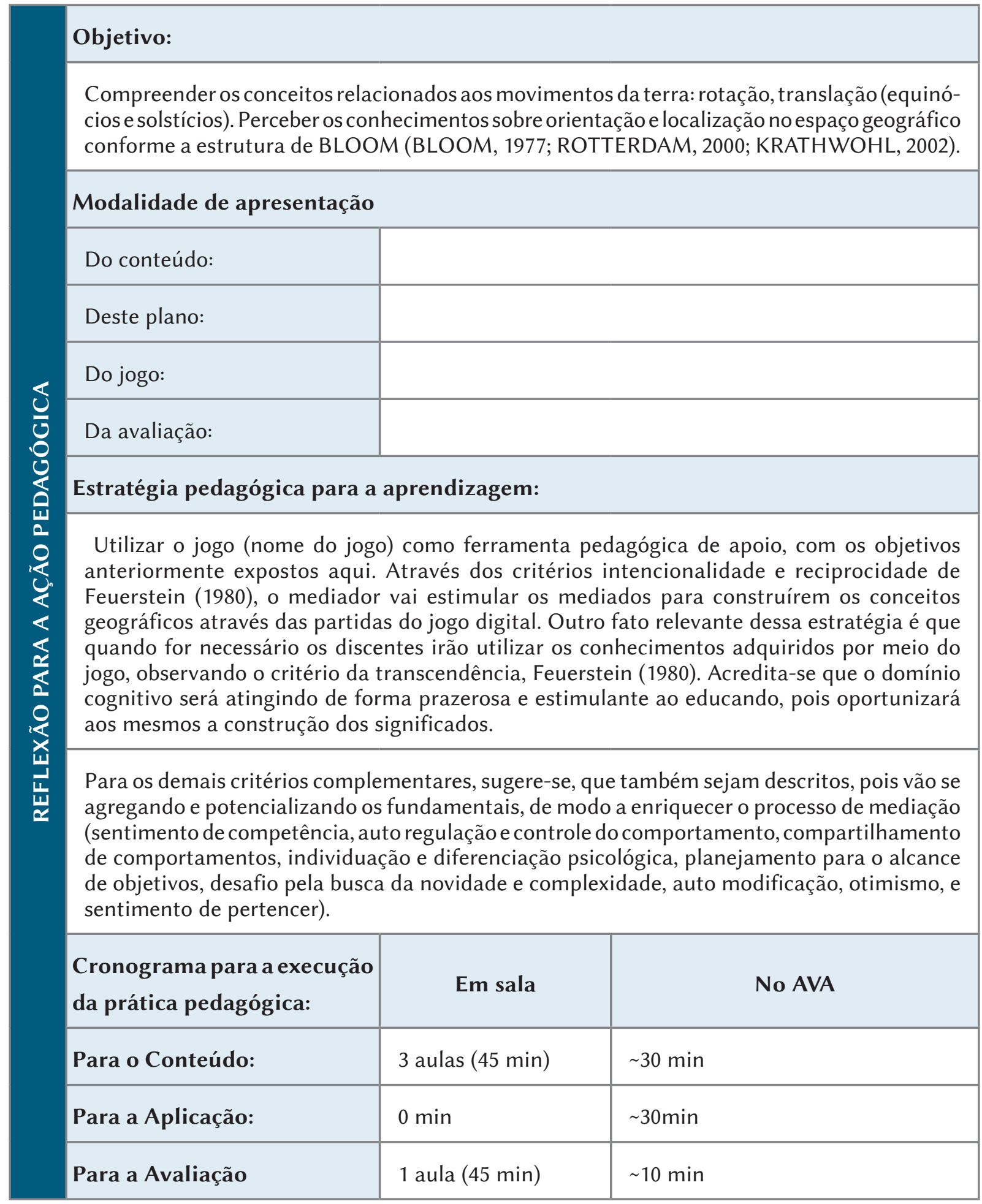


Espera-se que os discentes compreendam os movimentos de rotação, translação, solstícios e equinócios e como seus resultados, dia, noite, ano estações do ano, interferem na interação com o espaço geográfico. Descrever os indicadores de aprendizagem de forma alinhada com objetivos pedagógicos e com os instrumentos de avaliação.
- Três questionários para avaliação do jogo e da aprendizagem

- Avaliação do conhecimento no conteúdo (pré e pós prática pedagógica)

- Avaliação da percepção do usuário sobre o jogo (imersão, desafio, diversão, outros); (pós prática).

- Avaliação por observação participante do mediador (registros na reflexão sobre a ação)

\section{Elementos relevantes durante a ação pedagógica:}

Acessos ao AVA, Compreensão, Facilidade de uso e Participação no fórum.

Fonte: Dos autores (2016)

\subsection{A definição do Jogo}

A partir de uma pesquisa relacionada ao tema do conteúdo programático, cerca de seis opções foram discutidas com o docente que optou pelo uso de dois jogos, conforme Tabela 1:

Tabela 1: Jogos definidos para a aplicação

\begin{tabular}{c|c|c}
\hline JOGO & DESENVOLVEDOR & TIPO \\
\hline $\begin{array}{c}\text { Game 01: } \\
\text { "Capitão Tormenta e Paco em: } \\
\text { Movimentos da Terra" }\end{array}$ & $\begin{array}{c}\text { RIVED (Rede Internacional } \\
\text { Virtual de Educação) / } \\
\text { Secretaria de Educação a } \\
\text { Distância (SEED) do MEC. }\end{array}$ & Charadas \\
\hline Game 02: & ClassTools.net & Questionário em árcade. \\
\hline
\end{tabular}

Fonte: Dos autores (2016)

Os jogos foram desenvolvidos em Adobe Flash $^{\circledR}$. Esse formato permite a integração no Moodle em um recurso de página por HTML (HyperText MarkupLanguage).Cada jogo possui uma URL (Uniform Resource Locator), que é então embarcada pela marcação "<embedded>”. Nesse caso, não é necessário carregar arquivos para o servidor, basta direcionar para a ligação do jogo no site original.
O Game 01 (Figura 3), apesar da arte gráfica mais elaborada e conteúdo pedagógico mais completo, apresentou pouca interação, pois possui apenas duas charadas.

Ao responder corretamente as duas questões, o usuário é convidado a subir a participar da tripulação do navio pirata e finaliza o jogo. 
Figura 3: Captura de tela do Game 01 embutido no Moodle GEOGRAFIA - $9^{\circ}$ ANO
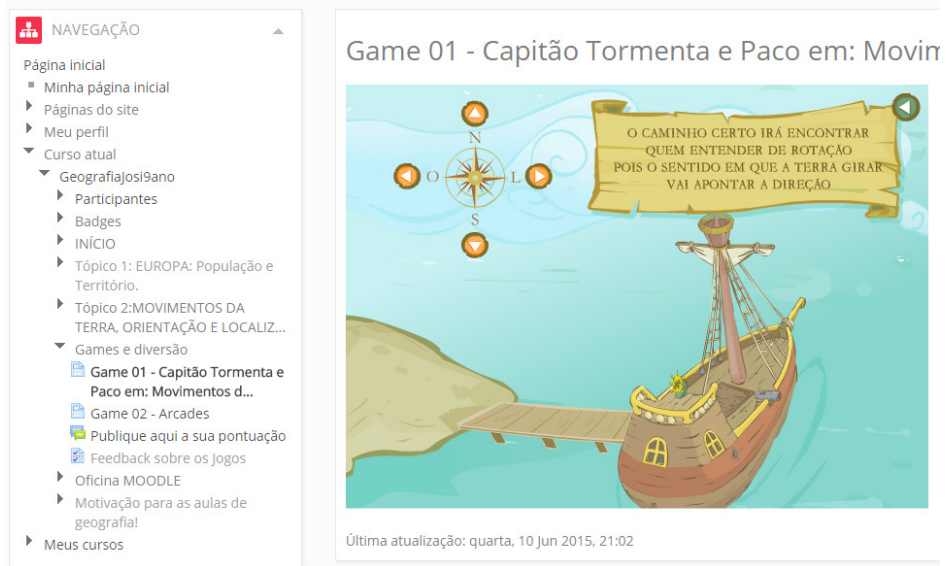

Fonte: Dos autores (2016)

Já o Game 02 (Figura 4), mesmo com a interface mais simples, possui diferentes níveis de dificuldade e maior interação. As questões podem ser programadas e ampliadas, abrindo uma oportunidade de maior uso, além de permitir a edição.

A fim de criar uma competição, os estudantes foram incentivados a publicar suas pontuações em um fórum disponibilizado também no Moodle.

Figura 4: Captura de tela do Game 02 embutido no Moodle
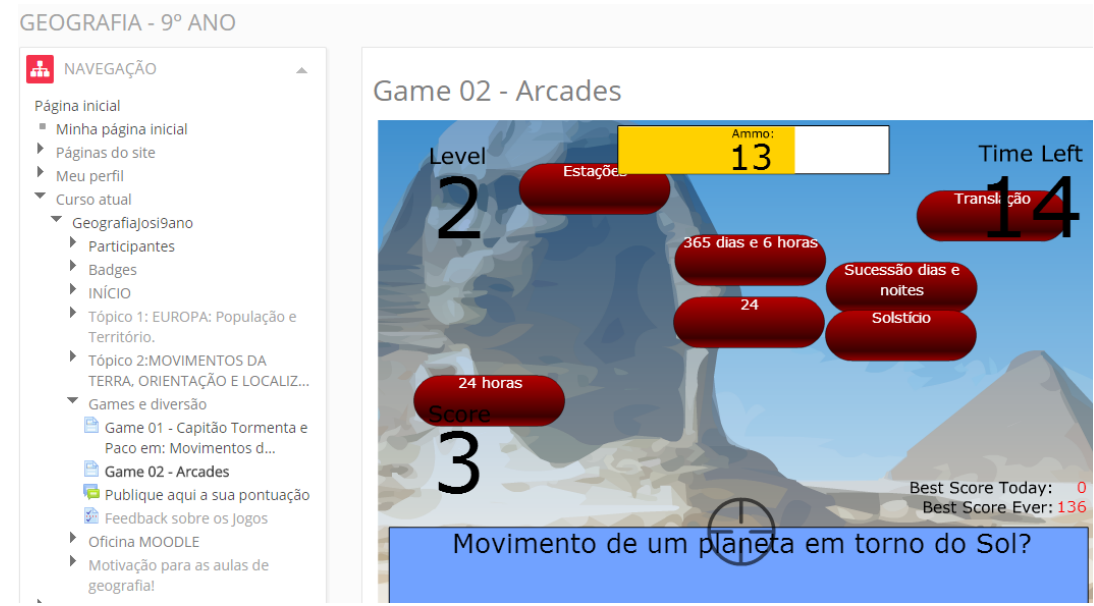

Fonte: Dos autores (2016)

\section{RESULTADOS E DISCUSSÕES}

Para essa pesquisa, foram acompanhados os estudantes da $9^{\circ}$ ano do Ensino FundamentalII. A turma participou de uma pré-análise definida como avaliação diagnóstica sobre o conteúdo de movimentos da Terra e discutiram em sala de aula sobre o conteúdo.
Nessa pré-análise, a docente observou que os estudantes possuíam um breve conhecimento dos conceitos, porém não os compreendiam na totalidade. Por exemplo, lembravam do termo rotação, mas não das suas consequências. 
Os jogos foram disponibilizados por duas semanas no Moodle, AVA que está em período de implantação e testes pela docente há um mês. No primeiro contato dos estudantes com o AVA, houve problemas no acesso devido ao desconhecimento da plataforma, o que acarretou em poucos acessos. Julgou-se então necessária a realização de uma Oficina na sala de informática da escola.

AOficina foi realizada na $1^{\mathrm{a}}$ semana e, por questão de disponibilidade de horários, ocorreu após a última aula do período letivo. Apesar disso, $89 \%$ dos estudantes da turma participaram. No treinamento aprenderam a acessar o ambiente, publicar tarefas e a responder a questionários, além de explicar a atividade disponibilizada no AVA.

\footnotetext{
Em consequências das limitações da banda larga de apenas $1 \mathrm{Mbit} / \mathrm{s}$, os estudantes não puderam praticar.
}

Porém, foram instigados a preencher a enquete "Perfil dos discentes" e a participar dos jogos em outro período, quando tivessem acesso à internet.

\subsection{Perfil dos discentes}

A enquete "Perfil dos discentes" houve grande participação: dos 19 estudantes matriculados, 15 responderam à enquete, ou seja, cerca de $80 \%$ da turma.A idade desses participantes está na faixa entre 13 e 16 anos, com média de 14,5 anos. Todos possuem smartphone, celular ou tablet, sendo que $40 \%$ não possuem computadores ou notebooks. Portanto, os dispositivos móveis são mais comuns do que os computadores pessoais.
Sobre o uso da internet, cerca de $80 \%$ passa mais do que uma hora conectado por dia. Em relação às atividades que fazem nesse período, apenas 40\% utilizam a internet para jogar, número inferior até mesmo aos $67 \%$ que indicaram que utilizam o serviço para estudar. Porém, $80 \%$ indicaram que gostam de jogos digitais (4 a 5 na escala lickert $^{2}$ ) e apenas $20 \%$ indicaram que não gostam ou não têm interesse em jogos digitais (1 a 3 na escala lickert).

Ainda em relação ao gosto por jogos digitais e AVA, a média na escala lickert foi de 4,07 para jogos digitais e 4,7 para o AVA. Ao qual cerca de $30 \%$ gostam mais do AVA e $13 \%$ preferem os jogos.

\section{ESSA CONSTATAÇÃO MOSTRA A}

IMPORTÂNCIA DOS AMBIENTES

VirTUAIS DE APRENDIZAGEM,

TALVEZ POR INflUÊNCIA DA

OficINA QUE MOSTROU AS

FACILIDADES DO AMBIENTE NA

REALIZAÇÃO DOS EXERCÍCIOS

E ENTREGA DE TAREFAS, POR

EXEMPLO.

\subsection{Avaliação do artefato}

Para avaliar o artefato, nesse caso, o plano de aplicação desenvolvido, foram analisadas as enquetes, as participações e a visão da docente. O Quadro 3 apresenta os principais requisitos considerados em relação ao plano de aplicação e aos objetivos desse artigo.

\footnotetext{
2 Técnica de atribuição em escala quantitativa de 1 a 5 (LIKERT, 1932).
} 
Quadro 3: Requisitos de Avaliação em relação ao artefato

\begin{tabular}{|c|c|c|c|}
\hline Organização do Plano & SIM & $\begin{array}{c}\text { Adequado para a organização e reflexão da ação } \\
\text { como um complemento ao plano de aula. }\end{array}$ \\
\cline { 2 - 4 } & $\begin{array}{c}\text { Características } \\
\text { Tecnológicas }\end{array}$ & SIM & $\begin{array}{c}\text { Bastante completo, porém há requisitos que o } \\
\text { docente necessita de uma ajuda mais técnica. }\end{array}$ \\
\hline $\begin{array}{c}\text { Características } \\
\text { Pedagógicas }\end{array}$ & SIM & De acordo com as necessidades apresentadas. \\
\hline Facilidade de uso & SIM & $\begin{array}{c}\text { De acordo desde que complementado com o } \\
\text { plano de aula comum. }\end{array}$ \\
\hline
\end{tabular}

Fonte: Adaptado de Dresch et al. (2015)

O Quadro 4 apresenta os requisitos considerados para avaliação da ação, nesse caso, a aplicação dos jogos digitais a Distância, que foi mediada através do AVA Moodle. Apesar do planejamento organizado e descrito no plano apresentado, estas reflexões apresentam as limitações e características a serem melhor compreendidas ou descritas no planejamento e disponibilização das próximas atividades com os jogos digitais a Distância.

Quadro 4: Requisitos de Avaliação em relação a ação

\begin{tabular}{|c|c|c|c|}
\hline 응 & Acessos ao AVA & SIM & $\begin{array}{l}\text { Durante o período de aplicação (2 semanas), todos os estu- } \\
\text { dantes tiveram acesso ao menos semanal no ambiente. }\end{array}$ \\
\hline$\stackrel{0}{2}$ & Compreensão & SIM & $\begin{array}{l}\text { O relato dos próprios estudantes foi: "Depois dos jogos } \\
\text { é impossível não lembrarmos dos conceitos de rotação e } \\
\text { translação". }\end{array}$ \\
\hline 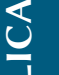 & Facilidade de uso & NÃO & A extensão dos jogos não permitiu o uso multiplataforma. \\
\hline$\frac{2}{\alpha}$ & $\begin{array}{l}\text { Participação no } \\
\text { fórum }\end{array}$ & NÃO & $\begin{array}{l}\text { Poucos estudantes, cerca de } 30 \% \text { da turma, publicaram as } \\
\text { pontuações no fórum disponibilizado. }\end{array}$ \\
\hline
\end{tabular}

Fonte: Adaptado de Dresch et al. (2015)

\subsection{Explicitação das aprendizagens}

Os estudantes foram instruídos de que a atividade se tratava de uma atividade de pesquisa e seria necessário seguir as etapas solicitadas pelo docente. Apesar disso, a participação no fórum foi considerada baixa, com cerca de 30\% em duas semanas de aplicação. Porém, as demais informações foram suficientes para as constatações necessárias nessa pesquisa.

Vale ressaltar que não foi atribuída nota ou qualquer recompensa aos participantes, sendo os jogos considerados apenas como uma atividade complementar,conforme o Plano de Aplicação apresentado na Seção 4. Logo, outras formas 
de motivação ou mesmo pontuações extras poderiam incentivar mais participações.

Por fim, o plano de aplicação se mostrou adequado e preciso nas reflexões necessárias para a ação, não apenas nas explicitações pedagógicas, mas também tecnológicas e do conteúdo.

\section{LOGO, A INTEGRAÇÃO AO}

AVA NÃO PREJUDICOU E NÃO

TOMOU O TEMPO DO PERÍODO

LETIVO, DISPONIBILIZANDO UM

CONTEÚDO COMPLEMENTAR

AOS ESTUDANTES. dispositivo móvel, uma vez que, apesar dos números bastante positivos de acesso ao AVA e da participação nas enquetes aqui apresentadas, poucos estudantes puderam participardos jogos e fóruns devido à limitação da tecnologia.

A limitação pela tecnologia se deve ao fato de que os jogos disponibilizados foram desenvolvidos em Adobe Flash ${ }^{\circledR}$. Essa linguagem está em desuso e obsoleta para dispositivos móveis e, mais recentemente, também desabilitada nas versões mais recentes dos principais navegadores, como Google Chrome, Firefox e Safari. Portanto,não há mais suporte multiplataformas de objetos com a extensão Shockwave Flash (.swf). Logo, apesar do desenvolvimento nessa extensão permitir a integração no Moodle, a tecnologia não possui suporte nos dispositivos móveis, o que limita a participação a Distância dos estudantes que apenas acessam a internet através desses dispositivos.

Esse fato é preocupante, em especial pela quantidade de aplicativos e materiais com essa extensão disponibilizados no Banco Internacional de Objetos Educacionais $(\mathrm{BIOE})^{3}$. importante foi a escolha do AVA Moodle, por ser responsivo e manter uma organização linear dos conteúdos.

Além disso,éfundamental que a enquete "Perfil dos discentes" a "Avaliação Diagnóstica"sejam disponibilizadas no AVA e realizadas antes ou durante o período de planejamento da aplicação. Além disso, diversos requisitos influenciam diretamente na aplicação, entre eles podem ser citados: os tipos de dispositivos que os estudantes têm acesso, o período e o tipo de conexão à internet que possuem.

Assim, foi possível constatar como a limitação da tecnologia impossibilitou os estudantes em acessarem e realizarem a atividade através do
Em resposta à solicitação pelo e-SIC (SEED/ MEC,2015), a instituição apontou um número total de 19.842 objetos, dos quais 15.356 , ou seja, $73,53 \%$ dos objetos estão no formato “.swf”. São materiais riquíssimos que já sofrem as limitações da linguagem utilizada no desenvolvimento.

Cada vez mais serão imprescindíveis os requisitos de multiplataforma e design responsivo multitelas. No contexto educacional brasileiro, essas características são ainda mais primordiais, devido ao acesso às tecnologias ocorrer gradualmente e a partir de dispositivos de baixo custo que implicam processamento limitado,

\footnotetext{
${ }^{3}$ Disponível em http://objetoseducacionais2.mec. gov.br/
} 
conexão com a internet restringida e variedade de sistemas, telas e recursos.

\subsection{Generalização}

A pesquisa por soluções direcionadas a problemas específicos é uma orientação fundamental da design science. Apesar dessa busca não ser caracterizada como a solução ótima, mas por uma solução satisfatória, esses resultados necessitam ser generalizadas para outros contextos, definidos como classe de problemas (DRESCH et al., 2015).

A generalização para uma classe de problemas amplia as oportunidades para que outros pesquisadores e profissionais sejam capazes de utilizar o conhecimento gerado pela pesquisa em contextos diversos. Ao generalizar o conhecimento produzido, o objetivo é então obter um resultado desejado em um determinado contexto, a fim de também aplicá-lo em outros (VON ALAN et al., 2004).

Nesse caso, o aproveitamento das tecnologias tem proporcionado oportunidades da Educação Básica ao Ensino Superior, em qualquer modalidade, seja presencial ou a Distância. Atualmente há uma perspectiva do uso de ambientes imersivos, como mundos virtuais $3 \mathrm{D}$ e realidade aumentada (JOHNSON etal.,2014). Essas tendências já apresentam aplicações interessantes a serem exploradas.

Portanto, As CONTRIBUições

DESSA PESQUISA PERMITEM A

GENERALIZAÇÃO PARA OUTRAS

DISCIPLINAS E CONTEXTOS

DIFERENCIADOS. AfinAL, A

ADEQUADA ORGANIZAÇÃO É

SEMPRE ESSENCIAL AO SUCESSO

DA AÇÃO PEDAGÓGICA, O QUE

JUSTIfICA A NECESSIDADE DE

UM PROCESSO E PLANO COMO O

AQUI APRESENTADO.

Dentre as possibilidades observadas, a integração das disciplinas também colaboraria para facilitar que o estudante observe diferentes conceitos e saiba integrá-los, em um processo de aprendizagem interdisciplinar.

\section{CONSIDERAÇÕES FINAIS}

A reflexão sobre os critérios necessários permitiu prever as necessidades dos recursos e o envolvimento dos conhecimentos tecnológicos, pedagógicos e do conteúdo. A partir dos critérios levantados, a real execução foi essencial para perceber a importância dessa organização prévia, o que garantiu uma aplicação fácil e sem problemas maiores. A alternativa de disponibilizar os jogos no AVA garantiu a liberação de acesso aos estudantes de um conteúdo complementar e interessante para o aprendizado, sem prejudicar o tempo do período letivo. Outras observações e limitações mostraram ainda a necessidade de um estudo prévio do perfil discente. A pesquisa ainda discutiu a atenção necessária no desenvolvimento de jogos e objetos educacionais em linguagens compatíveis com os dispositivos móveis.

Em relação à metodologia adotada, os processos da Design Science Research foram adequados para o desenvolvimento do plano de aplicação 
e no levantamento dos critérios, colaborando na solução da questão de pesquisa. Com isso, os objetivos específicos de descrever, desenvolver e integrar foram estabelecidos, em especial com a organização do TPACK.

O TPACK requer uma percepção de conceitos e técnicas pedagógicas para integrá-los de forma construtiva ao ensino. Assim, é papel da reflexão com o TPACK responder aos estímulos tecnológicos e auxiliar na construção do conhecimento dos estudantes.

A partir dos resultados, é possível observar pontos planejados bastante positivos, como o acesso e o compartilhamento da pontuação no fórum. Porém, há também os pontos negativos observados em relação aos requisitos "Facilidade de uso" e "Participação no fórum", que não obtiveram resultados satisfatórios. Assim, as próximas ações devem melhor delimitar os jogos digitais e os recursos tecnológicos disponíveis, além de permitir o acesso ampliado aos estudantes na questão de tempo e também aplicação multiplataforma.

A partir disso, como trabalhos futuros, podem ser apontadas as necessidades de novas formas de avaliação, de incentivo aos estudantes e de relação interdisciplinar dos conteúdos. Novos estudos empíricos podem ainda demonstrar os impactos dos diferentes contextos sociais e indicar outras limitações. Portanto, dados relacionando grupos de estudantes são interessantes trabalhos para pesquisas futuras, a fim de adequar o plano nos diferentes contextos. Os materiais continuarão disponíveis aos estudantes e o interesse dos estudantes animou a docente a continuar utilizando o ambiente como recurso extra para as aulas.

\section{AGRADECIMENTOS}

Conselho Nacional de Desenvolvimento Científico e Tecnológico (CNPq) Chamada Universal - MCTI/CNPq No 14/2014.
Este projeto é financiado com recursos do Ministério da Educação (CAPES) como parte das ações de P\&D no escopo do Programa Interministerial da RNP. 


\section{DISTANCE LEARNING IN BASIC EDUCATION: A PEDAGOGICAL INTEGRATION OF DIGITAL GAMES IN VIRTUAL ENVIRONMENTS}

\begin{abstract}
The use of technology in education requires adequate integration of knowledge in order to avoid frustration and waste of time. Thus, an implementation plan needs to outline the reflections not only teaching, but also technological and content, a proposal for knowledge management related to TPACK (Technological Pedagogical Content Knowledge). The objective of this paper is to present the development of a game implementation plan that allows ample notice of the knowledgenecessary tocarryouteducationalactivities based on the distance TPACK. Thus, the plan allows systematize the integration of games in a pedagogical concept of extension of the classroom and the broadening of knowledge exposed. In addition, the analysis in an empirical study with a group of elementary school allowed validate the plan. Despite the limitations on the extensions of the available games, distance education proved feasible in addition to the content.
\end{abstract}

Keywords: Educational Games;

Geography Teaching; Virtual

Learning Environments;

Educational Objects.

\section{REFERÊNCIAS}

ALEXANDRE, M. I. et al. Impacts and barriers of the mobile remote experimentation introduced in basic education. Remote Engineering and Virtual Instrumentation (REV), 2014 11th International Conference on, 2014, 26-28 Feb. 2014. p.324-325.

BRASIL. Decreto no 1.917, de 27 de maio de 1996. Estrutura Regimental e o Quadro Demonstrativo dos Cargos em Comissão e Funções Gratificadas do Ministério da Educação e do Desporto e dá outras providências., 1996. Disponível em: < http://www.planalto.gov.br/ ccivil_03/decreto/1996/D1917.htm >. Acesso em: 20 de Junho de 2015.

. Parâmetros Curriculares Nacionais: apresentação dos temas transversais. MEC/SEF. Brasília. 1997

. Decreto no 5.622, de 19 de dezembro de 2005. Regulamenta o art. 80 da Lei no 9.394, de 20 de dezembro de 1996, que estabelece as diretrizes e bases da educação nacional. 2005. Disponível em: < http://www.planalto.gov.br/ccivil_03/_ato20042006/2005/decreto/d5622.htm >. Acesso em: 20 de Junho de 2015.

CAVALCANTI, L. D. S. Geografia, escola e construção de conhecimentos. Vol. 16. Campinas: Papirus Editora, 2010. ISBN 853080516X.

DRESCH, A.; LACERDA, D. P.; JÚNIOR, J. A. V. A. Design Science Research: Método de Pesquisa para Avanço da Ciência e Tecnologia. Bookman Editora, 2015. ISBN 8582602995.

GADOTTI, M. Perspectivas atuais da educação. São Paulo em perspectiva, v. 14, n. 2, p. 03-11, 2000. ISSN 0102-8839.

HUIZINGA, J. Homo Ludens Ils 86. Taylor \& Francis, 2014. ISBN 9781317834045.

Disponível em: < https://books.google.com.br/ books?id=HabrAgAAQBAJ > . 
INEP. Censo Escolar da Educação Básica - Resumo Técnico. Instituto Nacional de Estudos e Pesquisas Educacionais Anísio Teixeira 2013.

JANSSEN, N.; LAZONDER, A. Implementing Innovative Technologies Through Lesson Plans: What Kind of Support Do Teachers Prefer? Journal of Science Education and Technology, p. 1-11, 2015/06/30 2015. ISSN 1059-0145. Disponível em: < http://dx.doi.org/10.1007/s10956-015-9573-5 >.

JAPPUR, R. F. Modelo conceitual para criação, aplicação e avaliação de jogos educativos digitais. 2014. Universidade Federal de Santa Catarina

JOHNSON, L. et al. NMC horizon report: 2014 K. 2014. ISSN 0991482859.

KOEHLER, M.; MISHRA, P. What is technological pedagogical content knowledge (TPACK)?

Contemporary issues in technology and teacher education, v. 9, n. 1, p. 60-70, 2009. ISSN 1528-5804.

LIKERT, R. A technique for the measurement of attitudes. Archives of psychology, 1932.

MACHADO, D.; REIS, P. C.; BENTES, R. D. F. A evolução da educação à distância brasileira: análises da interação nos jogos e na rede social a partir do estudo de caso da universidade positivo. 2015.

MCDONALD, J. Exam Review Strategies. 2004. Disponível em: < www.wlu.ca/documents/107/Exam_ Review_Strategies_Packages.pdf $>$.

MURTHY, S.; IYER, S.; WARRIEM, J. ET4ET: A Large-Scale Faculty Professional Development Program on Effective Integration of Educational Technology. Journal of Educational Technology \& Society, v. 18, n. 3, p. 16-28, 2015. ISSN 1176-3647.

PEFFERS, K. et al. A design science research methodology for information systems research. Journal of management information systems, v. 24, n. 3, p. 45-77, 2007. ISSN 0742-1222.
PEREIRA, A. T. C.; SCHMITT, V.; DIAS, M.

Ambientes virtuais de aprendizagem. AVA-Ambientes Virtuais de Aprendizagem em Diferentes Contextos. Rio de Janeiro: Editora Ciência Moderna Ltda, 2007.

PEREIRA, R. S. A Reflexão e a Prática no Ensino: Geografia. Ed.07. São Paulo: 2012.206 ISBN 9788521206637.

ROZA, L. A. G. Freud e o inconsciente. Rio de Janeiro: Jorge Zahar, 1996.

SALEN, K.; ZIMMERMAN, E. Regras do JogoFundamentos do Design de Jogos. vol. 1, 2, 3 and 4. Editora Blucher, São Paulo, 2012.

SECRETARIA DE EDUCAÇÃO A DISTÂNCIA - SEED. Relatório de Gestão. Ministério da Educação. Brasília/DF. 2009

SEED/MEC. Banco Internacional de Objetos Educacionais (BIOE). 2015. Disponível em: < http:// objetoseducacionais2.mec.gov.br/ >.

SHULMAN, L. Knowledge and teaching: Foundations of the new reform. Harvard educational review, v. 57, n. 1, p. 1-23, 1987. ISSN 0017-8055.

THOMPSON, J.; BERBANK-GREEN, B.; CUSWORTH, N. Game Design: Principles, Practice, and Techniques-The Ultimate Guide for the Aspiring

Game Designer. John Wiley \& Sons, 2007. ISBN 0471968943.

VON ALAN, R. H. et al. Design science in information systems research. MIS quarterly, v. 28, n. 1, p. 75-105, 2004.

Data de recebimento: 28/09/2015

Data de aprovação: 29/07/2016 


\section{SOBRE OS AUTORES}

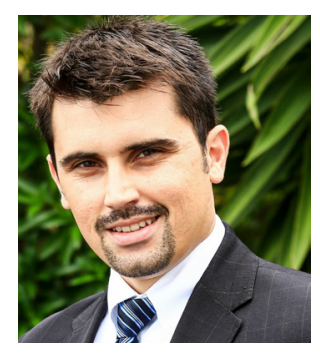

\section{Willian Rochadel}

É doutorando e mestre no Programade Pós-Graduação em Engenharia e Gestão do Conhecimento (PPGEGC) da Universidade Federal de Santa Catarina (UFSC), com estudos na área de avaliação e seleção de ideias para Inovação no Grupo de Pesquisa IGTI (Inteligência, Gestão e Tecnologia para Inovação). Atua no Laboratório de Experimentação Remota (RExLab) como pesquisador em sistemas embarcados e IoT para educação. Servidor Técnico Administrativo no Setor de TI da UFSC. Bacharel em Tecnologias da Informação e Comunicação (UFSC) e Técnico em Eletrotécnica pelo CEDUP Dario Geraldo Salles.

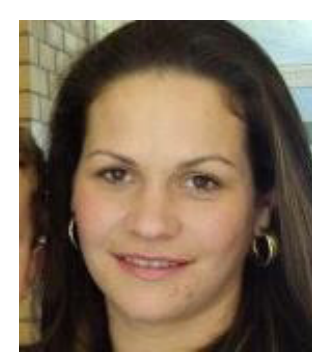

\section{Josi Zanette do Canto}

É mestranda no Programa de Pós-Graduação em Tecnologias da Informação e Comunicação (PPGTIC) da Universidade Federal de Santa Catarina (UFSC), cursa Especialização em Educação na Cultura Digital (UFSC), possui especialização em metodologia do ensino interdisciplinar, pelaFUCAP/SC (2010) egraduação emLicenciatura Plena em Geografia pelo Centro Universitário Leonardo Da Vinci/SC (2009). Atualmente é professora de geografia ehistória do ensino fundamental IIe Médio da rede estadual e particular no município de Araranguá/SC.Continuamente busca aintegração das tecnologias digitais com a sala de aula. Tem experiência na área de Licenciatura em Geografia, com ênfase em professor de ensino fundamental II, médio e educação de jovens e adultos.

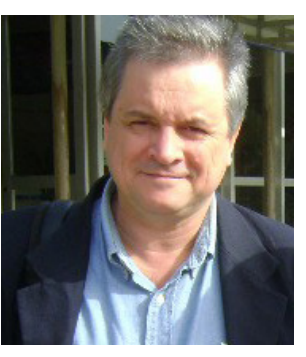

Juarez Bento da Silva

É graduado em Administração de Empresas pela Pontifícia Universidade Católica do Rio Grande do Sul (1991), mestre em

Ciências da Computação (2002) e doutor em Engenharia e Gestão do Conhecimento (2007) pela UFSC. Atualmente é professor adjunto da UFSC onde atua como docente nos cursos de graduação em Engenharia de Computação e Tecnologias da Informação e Comunicação e também no PPGTIC. Tem experiência nas áreas de Computação e TIC, com ênfase em Hardware, atuando, principalmente, nos seguintes temas: experimentação remota,e-learning,mre-learning,m-learning,mundos virtuais 3D, ambientes virtuais de ensino-aprendizagem, embedded serverse integração de tecnologias em disciplinas da Educação Básica na rede pública de ensino. É coordenador do Laboratório de Experimentação Remota (RExLab).

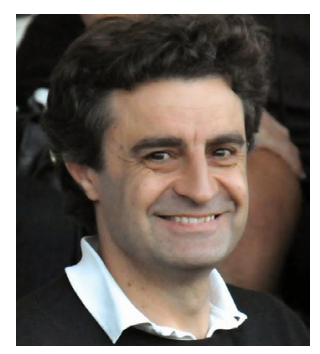

\section{Manuel A. González Delgado}

É graduado em Física pela Universidade de Valladolid (Espanha) e PhD em Física pela mesma universidade. Tem colaborado e publicado com pesquisadores da Universidade de Marselha, do Instituto Kurchatov de Moscou, do Instituto de Física de Belgrado, do Instituto Weizmann de Rehovot (Israel) e da Universidade da Flóridaem Gainesville,entre outros. Em paralelo, tem também trabalhado no desenvolvimento de diferentes ferramentas para o ensino de física como laboratórios virtuais na Internet ou aplicativos móveis e ambientes móveis para aprender física, bem como o uso de dispositivos móveis para 
fazer experimentos em laboratórios de Física. Seu ensinamento tem sido desenvolvido principalmente em Escolas de Engenharia.

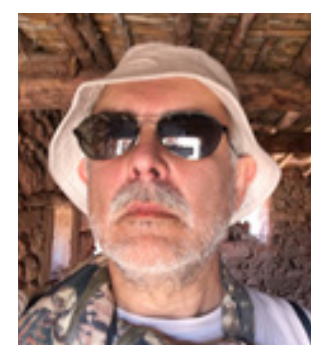

\section{Miguel A. González \\ Rebollo}

É graduado em Física pela

Universidade de Valladolid, na Espanha (1977) e doutor em Física pela mesma universidade (1983). De 1980 a 1982 foi bolsista de investigação na Universidade de Montpellier. Foi professor da Universidade de Marrakech de 1983 a 1985. E, atualmente, trabalha no grupo de pesquisa de semicondutores desenvolvendo novas técnicas para a caracterização microscópica e nanoscópica de semicondutores. O principal interesse na pesquisa agoraé o desenvolvimento de novas formas deutilizar dispositivos móveis em laboratórios de física.

\section{****}

